\title{
Immunotherapy in Prostate Cancer
}

\author{
(1) Deniz Bolat MD1,3, (1) Ayfer Haydaroğlu MD2,3 \\ 1 University of Health Sciences, Izmir Bozyaka Training and Research Hospital, Clinic of Urology, Izmir, Turkey \\ 2 Ege University Faculty of Medicine, Department of Radiation Oncology, Izmir, Turkey \\ 3 Ege University Institute of Health Sciences, Department of Basic Oncology, Izmir, Turkey
}

\begin{abstract}
In recent years, immunotherapy has become an important treatment alternative in the treatment of many cancers. Research on immunotherapy in prostate cancer has been accelerated by obtaining Food and Drug Administration (FDA) approval of sipuleucel-T for asymptomatic or minimal symptomatic metastatic castration-resistant prostate cancer (CRPC). Despite all these developments, the patients in whom these agents should be used, sequential use and combination strategies remain unclear. In this review, mechanisms of action and survival outcomes of different immunotherapeutic agents and therapeutic cancer vaccines in MCRPC are discussed.
\end{abstract}

Keywords: Prostate cancer, immunotherapy, vaccine, checkpoint blockers, survival

\section{Introduction}

In recent years, treatment alternatives for metastatic castrationresistant prostate cancer (MCRPC) have significantly increased and nowadays, many agents that have been proven to prolong overall survival in this population have been introduced. In addition to docetaxel, which is the backbone of the MCRPC chemotherapy, cabazitaxel provides an additional conventional approach. New generation antiandrogens targeting androgen inhibition such as enzalutamide and abiraterone offer a better toxicity profile. Radium-223 is aradiopharmaceutical and a unique option for patients with symptomatic bone metastasis.

The Food and Drug Administration (FDA) approval of sipuleucel-T in asymptomatic or minimally symptomatic MCRPC initiated the modern era of cancer immunotherapy.

In the progressive process, persistent improvements in survival with checkpoint blockers in patients with different solid tumors resulted in a change in treatment practices. However, although the response rates and survival benefits of checkpoint blockers in prostate cancer have been inadequate so far, the symptoms associated with clinical benefit suggest that these agents should not be abandoned. Strategic patient selection and tactical combination approaches can be a key to unlock immunotherapy in this disease.

\section{Checkpoint Inhibitors}

\section{CTLA-4 Inhibitors}

Ipilimumab is the first checkpoint inhibitor approved by the FDA in 2011. Ipilimumab is an antibody that blocks cytotoxic
T-lymphocyte antigen-4 (CTLA-4) and showed remarkable improvement in overall survival in advanced stage melanoma $(1,2)$. This drug, a complete human IgG monoclonal antibody, inhibits the binding of $B-7$ on antigen presenting cells (APC) with CTLA-4. Inhibition of CTLA-4/B-7 interaction reveals T cell activation and proliferation. Early ipilimumab clinical trial data from the MCRPC caught a glimpse of clinical activities in this population and provided a justification for additional research.

The first study evaluating Prostat specific ontigen (PSA) modulation and efficacy of ipilimumab in MCRPC was reported by Small et al. (3). As a result of this monotherapy pilot study, a decrease in PSA $>50 \%$ was observed in two patients for 135 days and 60 days, respectively. A decrease in PSA $<50 \%$ was reported in the remaining eight patients. Although PSA response is not a good indicator of radiographic response and clinical benefit, these improvements suggested that further evaluation of ipilimumab is needed.

There are two large phase III studies evaluating the effect of ipilimumab on survival in the MCRPC. In the first study, 799 patients with docetaxel-resistant prostate cancer and at least one bone metastasis were divided into $10 \mathrm{mg} / \mathrm{kg}$ ipilimumab and placebo groups after radiotherapy (4). The primary outcome of the study was overall survival (OS). In the ipilimumab arm, OS was 11.2 months and 10 months in the placebo arm (HR: 0.85, 95\% Cl: 0.72-1.00; $\mathrm{p}=0.053)$. Although this study did not meet the primary outcome, there was no OS benefit in the posthoc subgroup analyzes with poor prognostic factors in patients with visceral metastasis, high alkaline phosphatase or low hemoglobin levels, whereas OS benefit was found in the good prognostic group $(p=0.0038)$. This post-hoc analysis result

Address for Correspondence: Deniz Bolat MD, Health Sciences University, İzmir Bozyaka Training and Research Hospital, Clinic of Urology, İzmir, Turkey Phone: +90 5056383010 E-mail: drbolat@hotmail.com ORCID: orcid.org/0000-0001-7338-8737 Received: 18.09.2018 Accepted: 30.01.2019 
contributed to evidence that patients with good prognostic factors would benefit more from immunotherapy $(5,6,7)$.

In another phase III study, non-chemotherapy-treated asymptomatic or minimally symptomatic MCRPC patients without visceral metastasis were randomized to ipilimumab and placebo groups in a two: 1 ratio (8). Overall survival, the primary outcome, did not show a statistically significant difference between the two groups. The median OS was 28.7 months in the ipilimumab arm and 29.7 months in the placebo arm (HR: $1.11 ; 95 \% \mathrm{Cl}: 0.88-1.39 ; \mathrm{p}=0.3667)$. However, progression-free survival (PFS) was 5.6 months in the ipilimumab arm and 3.8 months in the placebo arm (HR: $0.67 ; 95.87 \% \mathrm{Cl}: 0.55-0.81$ ), while the PSA response was $23 \%$ in the ipilimumab arm and $8 \%$ in the placebo arm. Significant toxicities were identifiedand the most common side effects associated with the treatment were diarrhea, rash, itching, fatigue, nausea/vomiting and decreased appetite. In the ipilimumab arm, nine treatmentrelated deaths were reported, while no death was observed in the placebo arm. It is emphasized that this situation requires further research.

Tremelimumab, another anti-CTLA-4 agent, was evaluated in clinical trials of patients with different solid organ tumors. In 11 patients with prostate cancer with PSA recurrence, safety and PSA kinetics were evaluated following short-term androgen suppression treatment with tremelimumab (9). In this small population study, no change in PSA was observed. However, in three patients who had been on tremelimum for months following treatment, prolonged PSA doubling time was observed immediately after two doses. Although the PSA response with CTLA-4 inhibitors is interesting, further analysis is needed because of the hopeless results and the accompanying toxicity with ipilimumab monotherapy in prostate cancer.

\section{PD-1/PDL-1 Inhibitors}

FDA-approvedPD1/PDL-1 inhibitors, nivolumab, pembrolizumab, durvalumab, atezolizumab, and avelumab have so far been less pronounced in prostate cancer compared to the impressive results in other solid organ tumors.

In one of the first studies evaluating nivolumab in solid tumors including 17 prostate cancer patients, no objective response was reported (10). In the phase lb study in which pemrolizumab was evaluated in 23 patients with MCRPC and PDL-1 expression level $>1 \%$, partial response was observed in only three patients (11). The median response time was 59 weeks (28-62 weeks) and the overall response rate was $13 \%$ (95\% Cl: $3-34 \%)$. Although the response rate was moderate, the response time was promising.

In 18 patients with MCRPC in whom PDL-1 inhibitor avelumab was evaluated, no objective response could be obtained (12). However, in a small subgroup of five patients who received enzalutamide therapy with elevated PSA, three patients had stable disease lasting more than 24 months.

Clinical studies evaluating checkpoint blockers in prostate cancer have suggested that the use of these agents alone will result in less improvement than optimal in OS. However, these studies provide a perspective in terms of efficiency and should not be completely abandoned in this population. By combining with vaccines, hormonal agents or other modalities, further studies will help to understand the optimal approach to the use of checkpoint inhibitors in antitumor activity.

\section{Therapeutic Cancer Vaccines}

Sipuleucel-T showed improvement in OS in asymptomatic or minimally symptomatic MCRPC $(13,14)$. Ultimately, it was the first cancer-approved therapeutic cancer vaccine of the FDA. These studies that change the practice have shown that prostate cancer is susceptible to immunotherapy and vaccination treatment is an effective and safe approach.

\section{DCVAC/PCa}

DCAVAC/PCa is an autologous vaccine and contains activated dendritic cells stimulated with killed PSA-positive LNCaP cells. The combination of DCVAC/PCa with standard dose docetaxel and prednisone was evaluated in 25 patients with MCRPC in a phase I/II, open label, single-arm clinical trial (15). The primary and secondary outcomes of the study were identified as safety and immune responses. The most common side effects were fatigue, back pain and paresthesia (all of them were gr1 or 2). As part of the safety assessment, OS was compared to the predicted values through the previously developed nomograms. OS with DCVAC/PCa regimen was 19 months and this result was reported to be significantly longer than the 11.8 months and 13 months predicted in the Halabi and MSKCC nomograms (HR: $0.26,95 \% \mathrm{Cl}$ : 0.13-0.51).

The phase III study, VIABLE, is currently under way to further explore the potential of this promising treatment. VIABLE study was a randomized, double-blind, placebo-controlled, parallel group study and examined the efficacy and safety of placebo in 1200 patients with docetaxel + DCVAC/PCa versus docetaxel + placebo. The primary outcome is OS and the estimated end date of the study is June 2018 (16).

\section{PROSTVAC}

PROSVAC is a poxviral-based vaccine that encodes three co-stimulatory molecules (B7.1, ICAM-1 and LFA-3) together with PSA as the target antigen. In the phase II randomized, doubleblind study with 125 patients with MCRPC, the PROSTVAC prime-boost regimen showed significant improvement in OS $(17,18)$. The median OS with PROSTVAC was found to be 25.1 months and 16.6 months in the control arm (HR: $0.56,95 \% \mathrm{Cl}$ : 0.37-0.85; $p=0.0061$ ).

In another study, the immune effect induced by PROSTVAC administration in 104 patients was evaluated (19). T-cell responses were compared before and four weeks after vaccination. Overall, 59/104 patients (57\%) showed an increase in PSA-specific T-cell response, and 19/28 (68\%) patients were shown to develop immune responses to tumor-associated antigens that were not present in the patient, and this concept is known as antigen spread.

These promising results paved the way for phase III study called PROSPECT (20). PROSPECT study is a double-blind study in asymptomatic or minimally symptomatic 1297 MCRPC patients, and patients were randomized to the PROSTVAC, PROSTVAC + GM-CSF or placebo arms. In the interim evaluation 
conducted in September 2017, the primary outcome, OS, could not be reached and the study was terminated (21).

Although the results are disappointing, prospects for immunotherapy in prostate cancer may lie beneath the combination strategies. Studies on the combination of PROSTVAC with other immunotherapeutic agents or early cure of the disease continue.

\section{GVAX-PCa}

GVAX-PCa vaccine consists of cells derived from LNCap and PC3 cell lines and genetically modified to secrete GM-CSF. In a phase I/II dose escalation study performed on 80 patients with MCRPC, the vaccine was shown to be well tolerated and the most common side effect was erythema at the injection site (22). A significant proportion of $89 \%$ of the high dose group $(p=0.002)$ has been reported to have an antibody against one or two cell lines.

Two phase III studies evaluating safety and OS were completed. In the first study, docetaxel + prednisone was compared to GVAX in MCRPC patients without chemotherapy (23). The study was terminated early because it did not meet the primary outcome, OS. The median survival in 626 patients analyzed was 20.7 months in the GVAX arm and 21.7 months in the control arm (HR: 1.03, 95\% Cl: 0.83-1.28; $\mathrm{p}=0.78$ ). Grade III and above side effects were reported in $8.8 \%$ of the GVAX arm and in $43 \%$ of the docetaxel arm, and researchers reported that GVAX had a better toxicity profile. On the other hand, phase III study comparing GVAX + docetaxel with docetaxel alone in 408 MCRPC patients was terminated early due to imbalance in patient deaths (67 in vaccine group and 47 in docetaxel alone) (24). The imbalance was also reflected to the OS, and it was 12.2 months in the vaccine arm and 14.1 months in the chemotherapy arm $(p=0.0076)$. Further analysis is required on the subject.

\section{CV9104}

CV9103 is a MRNA vaccine that encodes PSA, PSCA, PSMA and STEAP1 antigens (25). In a phase I/lla study, 26 of 33 patients developed immune responses (25). OS was found to be significantly longer in patients who developed an immune response to multiple antigens than patients with no response or response to only one antigen (HR: $0.41,95 \% \mathrm{Cl}: 0.17-0.95$, $\mathrm{p}=0.017$ ).

Second generation CV9103 formulations also encode PAP and MUC1 antigens in addition to the former (26). In phase Ilb study, the improvement in primary outcome, OS, was not met in patients with asymptomatic or minimal symptomatic MCRPC (27).

\section{Combination Strategies}

The results regarding the effectiveness of the checkpoint inhibitors alone in the MCRPC have been disappointing so far. The mechanism behind this resistance must be clarified. Recent studies suggest that the tumor mutation load is predictive of a good response to PD1/PDL-1 $(28,29,30)$ and CTLA-4 inhibitors (31). Prostate cancer is known to have a low mutation load (32). For this reason, it would seem that further evidence would support this hypothesis. Interestingly, in one study, it was concluded that the results obtained following a PD-1/ PDL-1 inhibitor with CTLA-4 inhibitor were independent of the mutation load (28).

In a strategy, nivolumab with ipilimumab was evaluated in patients with advanced prostate cancer with androgen receptor mutation (33). As expected, a reduction of $50 \%$ PSA was achieved in one of 15 patients with accompanying toxicity, and in three out of 15 patients, persistent PFS was achieved (33).

\section{Vaccines and Checkpoint Inhibitors}

It is accepted that tumors with high PDL-1 expression in the tumor microenvironment tend to respond better to PD-1/ PDL-1 inhibitors (34). In a study by Rekoske et al., it was shown that PDL-1 expression was increased in circulating tumor cells following the PAP-encoding DNA vaccine, and it was thought that there was a relationship between PDL-1 up-regulation and PFS (35). The researchers also found a trend with the sipuleucel-T vaccine targeting PAP.

In the phase I study consisting of 30 MCRPC patients and evaluating the safety and tolerability of ipilimumab and PROSTVAC, the most reported side effects were injection site reaction, colitis, rash, elevation in aminotransferases and endocrine side effects (36). The median OS was 34.4 months for all patients and two-year OS was reported as $73 \%$. These results were found to be better than the previous vaccine alone and sipuleucel-T phase III studies. In particular, evidence has been obtained from this study for the spread of antigen by the immune response generated against tumor-associated antigens that are not present in the vaccine. Antigen spread may allow a more permanent and adaptive immune response that leads to improvement in long-term clinical outcomes (37).

In sipuleucel-T + ipilimumab study in nine patients, it was found that combination was well tolerated, and that postsipuleucel-T $\lg G$ and $\lg G-\operatorname{lgM}$ levels were increased for PAP ( $p<0.001$ and $p<0.0001$, respectively) and PA2024 ( $p=0.0001$ and $p<0.000$, respectively) compared to baseline levels. Furthermore, it was reported that IgG and IgG-IgM levels for PAP $(p<0.001$ and $p=0.002$, respectively) and PA2024 $(p<0.0001$ and $p=0.001$, respectively) increased from postsipuleucel-T to postipilimumab. OS, spuleucel-T and PA2024 and PAP-specific immune responses were previously evaluated and considered to have the potential for clinical benefit of the checkpoint vaccine regimen (38).

\section{Immunotherapy and Enzalutamide}

Enzalutamide competitively inhibits androgen binding, nuclear translocation of the androgen receptor and its interaction with DNA. Immunological characteristics of this second generation antiandrogen, which has the advantage of survival in MCRPC, have been characterized $(39,40)$. TRAMP mice were exposed to enzalutamide alone or in combination with the therapeutic vaccine by Ardiani et al. (41) and they reported increased thymic T-cell production and OS improvement in combination therapy compared to other therapies.

In the Phase II STRIDE study, 52 patients with MCRPC were randomized to either the concurrent or subsequent enzalutamide plus sipuleucel-T arms (42). According to the 
results of intermediate immuno-analysis, the PA2024-specific $\mathrm{T}$ cell response was increased in both arms $(\mathrm{p}<0.001)$ (43). In both arms, cytokines such as INF-gamma, TNF-alpha and IL-2 were increased. There was no difference in toxicity in concurrent and subsequent applications.

Bishop et al. (44) have shown that patients with progression under enzalutamide treatment have more PD-L1/2 positive dendritic cells than patients who respond to enzalutamide or who are enzalutamide-naive.

When pemrolizumab was administered to patients who were under enzalutamide treatment, more than 50\% PSA reduction was achieved in for out of 20 patients (45).

\section{Immunotherapy and Abiraterone}

Preclinical evidence suggests that abiraterone is also immunomodulatory, like enzalutamide (46). In a phase II study in 69 MCRCP patients comparing concurrent or subsequent abiraterone + prednisone and sipuleucel-T, the primary outcome was defined as cumulative antigen-presenting cell activation, and it has been shown that ex-vivo antigenpresenting cell activation and peripheral immune response increased in both arms compared to baseline $(p<0.05)(47)$. This study also showed that low-dose prednisone did not affect the immunogenicity of sipuleucel-T.

In the phase I/II study where the primary outcome was safety, the combination of abiraterone + prednisone with ipilimumab was evaluated (48). The study was terminated due to toxicities such as grade 3 hypokalemia, dehydration and transaminase elevation.

\section{Immunotherapy and PARP Inhibition}

Olaparib is a PARP inhibitor and shows clinical activity in patients with MCRPC and DNA repair defect (49). Mutations in DNA repair genes such as BRCA1/2, ataxia-telangiectasia, Fanconi anemiagenes, and CHEK2 are observed in 1/3 of the patients. In patients with treatment-resistant MCRPC, the effect of olaparib and durvalumab is evaluated in the ongoing single-arm pilot study (50). In the intermediate analysis, it was reported that the combination had an acceptable toxicity profile in 10 patients and a PSA decrease of more than $50 \%$ was observed in 5/7 (71\%) of the patients. Although the patient population has been less so far, the results of this study are particularly interesting given that they are given in an unselected population.

\section{Conclusion}

In large-scale studies other than sipuleucel-T, single-agent immunotherapies have not been shown to provide significant PFS and OS benefits in patients with MCRPC. The results of multiple phase III studies including ipilimumab and PROSTVAC were disappointing and revealed evidence that prostate cancer was not immunosensitive. However, benefit has been shown in smaller studies and it is thought that it is too early to abandon these agents completely. Determination of tumor and patient characteristics may be effective in response to immunotherapy. Combination strategies can overcome the escape from the immune response. The literature on avoiding the use of single-agent immunotherapy in MCRPC is increasing. Instead, resources should be concentrated on optimal patient selection and effective combinations to increase the immune response. Because therapeutic vaccines have a relatively low side-effect profile, research into their use in localized prostate cancer may be more valuable. Prostate cancer, showing biochemical recurrence, may be the optimal target population for immunotherapy regimens due to better toxicity profiles. Although checkpoint inhibitors are better tolerated than cytotoxic chemotherapies (51), these agents are associated with severe immune-mediated side effects. PD1/PDL-1 inhibitors are better tolerated than CTLA-4 inhibitors.

\section{Ethics}

Peer-review: Externally peer-reviewed.

\section{Authorship Contributions}

Consept: D.B., A.H.,Design: D.B., A.H., Data Collection and Processing: D.B.,Analysis and Interpretation: A.H., Literature Search: D.B., A.H., Writing: D.B.

Conflict of Interest: No conflict of interest was declared by the authors.

Financial Disclosure: The authors declared that this study received no financial support.

\section{References}

1. Eggermont AM, Chiarion-Sileni V, Grob IJ, et al. Prolonged survival in stage III melanoma with ipilimumab adjuvant therapy. N Engl J Med 2016;375:1845-1855.

2. Hodi FS, O'Day SJ, McDermott DF, et al. Improved survival with ipilimumab in patients with metastatic melanoma. $N$ Engl J Med 2010;363:711-723.

3. Small EJ, Tchekmedyian NS, Rini BI, et al. A pilot trial of CTLA-4 blockade with human anti-CTLA-4 in patients with hormone-refractory prostate cancer. Clin Cancer Res 2007;13:1810-1815.

4. Kwon ED, Drake CG, Scher HI, et al. Ipilimumab versus placebo after radiotherapy in patients with metastatic castration-resistant prostate cancer that had progressed after docetaxel chemotherapy (CA184-043): a multicentre, randomised, double-blind, phase 3 trial. Lancet Oncol 2014;15:700-712.

5. Gulley JL, Arlen PM, Madan RA, et al. Immunologic and prognostic factors associated with overall survival employing a poxviral-based PSA vaccine in metastatic castrate-resistant prostate cancer. Cancer Immunol Immunother2010;59:663-674.

6. Schellhammer PF, Chodak G, Whitmore JB, et al. Lower baseline prostate-specific antigen is associated with a greater overall survival benefit from sipuleucel-T in the Immunotherapy for Prostate Adenocarcinoma Treatment (IMPACT) trial. Urology 2013;81:1297-1302.

7. Gulley JL, Madan RA, Schlom J. Impact of tumour volume on the potential efficacy of therapeutic vaccines. Curr Oncol 2011;18:e150-e157.

8. Beer TM, Kwon ED, Drake CG, et al. Randomized, Double-Blind, Phase III Trial of Ipilimumab Versus Placebo in Asymptomatic or Minimally Symptomatic Patients With Metastatic Chemotherapy-Naive Castration-Resistant Prostate Cancer. J Clin Oncol 2017;35:40-47.

9. McNeel DG, Smith HA, Eickhoff JC, et al. Phase I trial of tremelimumab in combination with short-term androgen deprivation in patients with PSA-recurrent prostate cancer. Cancer Immunol Immunother 2012;61:1137-1147. 
10. Topalian SL, Hodi FS, Brahmer JR, et al. Safety, activity, and immune correlates of anti-PD-1 antibody in cancer. N Engl J Med 2012;366:2443-2454.

11. Hansen A, Massard C, Ott PA, et al. Pembrolizumab for patients with advanced prostate adenocarcinoma: preliminary results from the KEYNOTE-028 study. Ann Oncol 2016;27Suppl6:abstract 725PD.

12. Fakhrejahani F, Madan RA, Dahut WL, Karzai K, Cordes LM, et al. Avelumab in metastatic castration-resistant prostate cancer (mCRPC). J Clin Oncol 2017;35Suppl6:159.

13. Small EJ, Schellhammer PF, Higano CS, et al. Placebo-controlled phase III trial of immunologic therapy with sipuleucel-T (APC8015) in patients with metastatic, asymptomatic hormone refractory prostate cancer. J Clin Oncol 2006; 24:3089-3094.

14. Kantoff PW, Higano CS, Shore ND, et al. Sipuleucel-T immunotherapy for castration-resistant prostate cancer. N Engl J Med 2010;363:411-422.

15. Podrazil $M$, Horvath $R$, Becht $E$, et al. Phase $I / I$ clinical trial of dendritic-cell based immunotherapy (DCVAC/PCa) combined with chemotherapy in patients with metastatic, castration-resistant prostate cancer. Oncotarget 2015;6:18192-18205.

16. Beer TM, Vogelzang $N$, JiřinaBartůňková J, et al. Autologous dendritic cell immunotherapy (DCVAC/PCa) added to docetaxel chemotherapy in a Phase III trial (viable) in men with advanced (mCRPC) prostate cancer. J Immunother Cancer 2015;3Suppl2:P164.

17. Kantoff PW, Schuetz T], Blumenstein BA, et al. Overall survival analysis of a phase II randomized controlled trial of a Poxviral-based PSA-targeted immunotherapy in metastatic castration-resistant prostate cancer. J Clin Oncol 2010;28:1099-1105.

18. Kantoff PW, Gulley JL, Pico-Navarro C. Revised overall survival analysis of a phase II, randomized, double-blind, controlled study of PROSTVAC in men with metastatic castration-resistant prostate cancer. J Clin Oncol 2017;35:124-125.

19. Gulley JL, Madan RA, Tsang KY, et al. Immune impact induced by PROSTVAC (PSA-TRICOM), a therapeutic vaccine for prostate cancer. Cancer Immunol Res 2014;2:133-141.

20. Gulley JL, Giacchino JL, Breitmeyer JB, et al. Prospect: a randomized double-blind phase 3 efficacy study of PROSTVAC-VF immunotherapy in men with asymptomatic/minimally symptomatic metastatic castration-resistant prostate cancer. J Clin Oncol 2015;5:1509-1512.

21. Bavarian Nordic [Press Release]. Independent Data Monitoring Committee Recommends Discontinuation of Bavarian Nordic's Phase 3 Study of Prostvac in Metastatic Prostate Cancer. Available from: http://www.bavarian-nordic.com/investor/ news/news. aspx?news=5308. [Last accessed on 2017 Sep 14].

22. Higano CS, Corman JM, Smith DC, et al. Phase $1 / 2$ dose-escalation study of a GM-CSF-secreting, allogeneic, cellular immunotherapy for metastatic hormone-refractory prostate cancer. Cancer 2008;113:975-984.

23. Higano C, Saad F, Somer B, et al. A phase III trial of GVAX immunotherapy for prostate cancer versus docetaxel plus prednisone in asymptomatic castration-resistant prostate cancer (CRPC). Proc Am SocClin Oncol 2009;27suppl15S:abstract LBA150.

24. Small E, Demkow T, Gerritsen WR. A phase III trial of GVAX immunotherapy for prostate cancer in combination with docetaxel versus docetaxel plus prednisone in symptomatic, castration-resistant prosate cancer (CRPC). Cancer Sci 2009;100:1389-1396.

25. Kubler H, Scheel B, Gnad-Vogt $U$, et al. Self-adjuvanted mRNA vaccination in advanced prostate cancer patients: a first-in-man phase I/Ila study. J Immunother Cancer 2015;3:26.

26. Rausch S, Schwentner C, Stenzl A, et al. mRNA vaccine CV9103 and CV9104 for the treatment of prostate cancer. Hum Vaccinlmmunother 2014;10:3146-3152.

27. CureVac: Topline Results of Phase IIB Clinical Trial with CV9104, and RNAactive Prostate Cancer Vaccine. In. Presented at the 35th Annual J.P. Morgan Healthcare Conference San Francisco; 2017.
28. Goodman AM, Kato S, Bazhenova L, et al. Tumor mutational burden as an independent predictor of response to immunotherapy in diverse cancers. Mol Cancer Ther 2017; 16: 2598-2608.

29. Le DT, Uram JN, Wang H, et al. PD-1 blockade in tumors with mismatch-repair deficiency. N Engl J Med 2015; 372:2509-2520.

30. Rizvi NA, Hellmann MD, Snyder A, et al. Mutational landscape determines sensitivity to PD-1 blockade in non-small cell lung cancer. Science 2015;348:124-128.

31. Snyder A, Makarov V, Merghoub T, Yuan J, Zaretsky JM, et al. Genetic basis for clinical response to CTLA-4 blockade in melanoma. $\mathrm{N}$ Engl ] Med 2014; 371: 2189-2199.

32. Chalmers ZR, Connelly CF, Fabrizio D, et al. Analysis of 100,000 human cancer genomes reveals the landscape of tumor mutational burden. Genome Med 2017; 9:34.

33. Boudadi K, Suzman DL, Luber B, et al. Phase 2 biomarker-driven study of ipilimumab plus nivolumab (Ipi/Nivo) for ARV7-positive metastatic castrate-resistant prostate cancer (mCRPC). J Clin Oncol 2017; 35: abstract 5035.

34. Taube JM, Klein A, Brahmer JR, et al. Association of PD-1, PD-1 ligands, and other features of the tumor immune microenvironment with response to anti-PD-1 therapy. Clin Cancer Res 2014;20:5064-5074.

35. Rekoske BT, Olson BM, McNeel DG. Antitumor vaccination of prostate cancer patient elicits PD-1/PD-L1 regulated antigen-specific immune responses. Oncoimmunology 2016;5:e1165377.

36. Madan RA, Mohebtash M, Arlen PM, et al. Ipilimumab and a poxviral vaccine targeting prostate-specific antigen in metastatic castriation-resistant prostate cancer: a phase I dose-escalation trial. Lancet Oncol 2012;13:501-508.

37. Gulley JL, Madan RA, Pachynski R, et al. Role of antigen spread and distinctive characteristics of immunotherapy in cancer treatment. J Natl Cancer Inst 2017;109. [doi: 10.1093/jnci/djw261].

38. Sheikh NA, Petrylak D, Kantoff PW, et al. Sipuleucel-T immune parameters correlate with survival: an analysis of the randomized phase 3 cinical trials in men with castration-resistant prostate cancer. Cancer Immunol Immunother 2013; 62:137-147.

39. Scher HI, Fizazi K, Saad F, et al. Increased surivial with enzalutamide in prostate cancer after chemotherapy. N Engl J Med 2012; 367:1187-1197.

40. Beer TM, Armstrong AJ, Rathkopf DE, et al. Enzalutamide in metastatic prostate cancer before chemotherapy. $\mathrm{N}$ Engl J Med 2014;371:424-433.

41. Ardiani A, Farsaci B, Rogers C), et al. Combination therapy with a second-generation androgen receptor antagonist and a metastasis vaccine improves survival in a spontaneous prostate cancer model. Clin Cancer Res 2013;19:6205-6218.

42. Quinn DI, Petrylak DP, Pieczonka CM, Sandler A, DeVries T, et al. A randomized phase II, open-label study of sipuleucel-T with concurrent or sequential enzalutamide in metastatic castration-resistant prostate cancer (mCRPC). J Clin Oncol 2014;32 Suppl 15: abstract e16071 [10.1200/jco.2014.32.15_suppl.e16071].

43. Quinn DI, Drake CG, Dreicer R, et al. Immune response from STRIDE, a randomized, phase 2, open label study of sipuleucel-T (sip-T) with concurrent vs. sequential enzalutamide (enz) administration in metastatic castration-resistant prostate cancer (mCRPC). J Clin Oncol 2015;33Suppl15:abstract5040 [doi: 10.1200/ jco.2015.33.15_suppl.5040].

44. Bishop JL, Sio A, Angeles A, Roberts ME, et al. PD-L1 is highly expressed in enzalutamide resistant prostate cancer. Oncotarget 2015;6:234-242.

45. Graff JN, Alumkal JJ, Drake CG, et al. First evidence of significant clinical activity of PD-1 inhibitors in metastatic, castration resistant prostate cancer (mCRPC). Ann Oncol 2016;27Suppl6:7190.

46. Ardiani A, Gameiro SR, Kwilas AR, Donahue RN, Hodge JW. Androgen deprivation therapy sensitizes prostate cancer cells to T-cell killing through androgen receptor dependent modulation of the apoptotic pathway. Oncotarget 2014;5:9335-9348. 
47. Small EJ, Lance RS, Gardner TA, Karsh LI, Fong L, et al. A randomized phase II trial of sipuleucel-T with concurrent versus sequential abiraterone acetate plus prednisone in metastatic castration-resistant prostate cancer. Clin Cancer Res 2015;21:3862-3869.

48. Danila DC, Kuzel T, Cetnar JP, Rathkopf DE, Morris MJ, et al. A phase $1 / 2$ study combining ipilimumab with abiraterone acetate plus prednisone in chemotheraphy- and immunotheraphy-naive patients with progressive metastatic castration resistant prostate cancer (mCRPC). J Clin Oncol 2016;34Suppl15:abstract e16507.

49. Mateo J, Carreira S, Sandhu S, Miranda S, Mossop H, et al. DNArepair defects and olaparib in metastatic prostate cancer. $\mathrm{N}$ Engl J Med 2015;373:1697-1708.
50. Karzai F, Madan RA, Owens H, Hankin A, Couvillon A, et al. Combination of PDL-1 and PARP inhibition in an unselected population with metastatic castrate-resistant prostate cancer (mCRPC). J Clin Oncol 2017;35Suppl15:abstract5026.

51. Nishijima TF, Shachar SS, Nyrop KA, Muss HB. Safety and tolerability of PD-1/PD-L1 inhibitors compared with chemotherapy in patients with advanced cancer: a meta-analysis. Oncologist 2017;22:470-479. 